\title{
Comunidades de Machos de Euglossini (Hymenoptera: Apidae) em Matas Semidecíduas do Nordeste do Estado de São Paulo
}

\author{
José M. M. Rebêlo ${ }^{1}$ e Carlos A. Garófalo \\ ${ }^{1}$ Departamento de Patologia, Universidade Federal do Maranhão, Praça Madre \\ Deus, 2 - 65.025-560, São Luís, MA. \\ ${ }^{2}$ Departamento de Biologia, Faculdade de Filosofia, Ciências e Letras de Ribeirão \\ Preto, USP, Avenida Bandeirantes, 3900, 14.040-901, Ribeirão Preto, SP.
}

An. Soc. Entomol. Brasil 26(2): 243-255 (1997)

Communities of Male Euglossine Bees (Hymenoptera: Apidae) in Semideciduous Forests from Northeastern São Paulo State

\begin{abstract}
Seasonal changes in species richness, composition, and abundance of male euglossine bees were determined by attracting individuals with cineole, eugenol and vanillin. The study was conducted at Estação Experimental de Zootecnia (EEZ), Municipio of Sertãozinho and at Santa Carlota Farm, Itaoca Section (SI), Municipio of Cajuru, State of São Paulo. Male euglossine bees were captured once every two weeks for one year in each site from 7:00 A.M. to 5:00 P.M. Males of 10 and 14 species were captured at EEZ and SI, respectively. All species sampled at EEZ were also found at SI showing that the areas were similar. Eulaema nigrita Lepeletier, Euglossa pleosticta Dressler and Euglossa fimbriata Rebêlo \& Moure were the most abundant species in both areas, and they accounted for $80.3 \%$ and $90.6 \%$ of the males captured at SI and EEZ, respectively. In both areas the males were most abundant in autumn (53.8\% of the total population at EEZ and $46.3 \%$ at SI) and the least abundant in winter ( $0.7 \%$ at EEZ and $4.8 \%$ at SI). Species richness and bee abundance by season were not significantly correlated in both areas. Cineole was the most attractive bait ( 9 species and 58.7\% of the males captures at EEZ and 13 species and $71.5 \%$ of the males at SI) followed by eugenol and vanillin.
\end{abstract}

KEY WORDS: Insecta, diversity, phenology, chemical baits.

RESUMO - A composição, abundância e mudanças sazonais da fauna de Euglossini foram determinadas pela atração de machos por iscas de cineol, eugenol e vanilina. O estudo foi realizado em duas Reservas do Estado de São Paulo: Estação Experimental de Zootecnia (EEZ), município de Sertãozinho, e Fazenda Santa Carlota, Secção Itaoca (SI), município de Cajuru. As coletas foram realizadas a cada 15 dias, das 08:00 às 17:00h, durante um ano, em cada localidade. Machos de 10 e 14 espécies foram atraídos na EEZ e SI, respectivamente. Todas as espécies amostradas na EEZ ocorreram também na SI proporcionando um coeficiente de similaridade entre as áreas de 0,83. Eulaema nigrita Lepeletier, Euglossa pleosticta Dressler e Euglossa fimbriata Rebêlo \& Moure foram as espécies mais abundantes em ambas as áreas, correspondendo a $80.3 \%$ da amostra total na SI e 90,6\% na EEZ. Em ambas as 
áreas os machos foram mais abundantes no outono (53.8\% da amostragem da EEZ e $46.3 \%$ da SI) e menos no inverno (0.7\% da EEZ e $4.8 \%$ da SI). A abundância de machos e o número de espécies amostradas por estação não foram significantemente correlacionados. Cineol foi a isca mais atrativa tanto na EEZ (58,7\% do total de machos coletados e 9 espécies) como na SI (71,5\% dos machos e 13 espécies) seguido por eugenol e vanilina.

PALAVRAS-CHAVE: Insecta, abelhas, diversidade, fenologia, iscas-odores.

Os Euglossini, conhecidos como "abelhas das orquídeas", distribuem-se exclusivamente na região neotropical, apresentando maior diversidade nas zonas quentes e úmidas equatoriais (Moure 1967). Ambos os sexos são eficientes agentes polinizadores de muitas espécies florais (Janzen 1971); os machos coletam líquidos aromáticos das flores de orquídeas e de outras fontes florais e não florais (Dressler 1982, Williams 1982, Janzen et al. 1982, Ackerman 1983a, b, Pearson \& Dressler 1985, Rebêlo \& Garófalo 1991) e, provavelmente, os utilizam na síntese de feromônios sexuais (Williams \& Witthen 1983). Por esse motivo eles são facilmente atraídos por certos terpenóides e hidrocarbonetos aromáticos, sintéticos, análogos àqueles presentes nas fragrâncias florais (Hills et al. 1972). A utilização desses compostos, como iscas, tem auxiliado no conhecimento da fauna de diferentes áreas biogeográficas, revelando novas espécies (Kimsey 1987).

Estudos de comunidades de machos de Euglossini, com uso de iscas-odoríferas, visando a obtenção de informações sobre diversidade, abundância sazonal e preferência por iscas têm sido realizados nas florestas tropicais do Panamá (Ackerman 1983a, 1989, Roubik \& Ackerman 1987), Costa Rica (Janzen et al. 1982), Peru (Pearson \& Dressler 1985) e da Amazônia Central (Becker et al. 1991, Oliveira \& Campos 1995). Nas florestas subtropicais do Brasil, destacam-se os trabalhos realizados por Rebêlo (1990) e Rebêlo \& Garófalo (1991), no Estado de São
Paulo.

Neste trabalho estudou-se a fauna de Euglossini em fragmentos de matas semidecíduas, com o objetivo de conhecer a diversidade, abundância relativa, flutuação sazonal e associação das espécies com iscasodores.

\section{Material e Métodos}

Áreas de Estudo. O estudo foi realizado na Estação Experimental de Zootecnia (=EEZ), situada no Município de Sertãozinho, SP e na Fazenda Santa Carlota (=FSC), Secção Itaoca (=SI), situada no Município de Cajuru, SP.

A EEZ está localizada a $21^{\circ} 8^{\prime} \mathrm{LS}$ e $47^{\circ}$ 59' LW, a uma altitude média de $548 \mathrm{~m}$ e é drenada pelos córregos Boa Vista e Santo Antônio, sendo o limite sul marcado pelo Ribeirão da Onça. A área, com cerca de 2.264 ha, é recoberta por fragmentos de matas, remanescentes da Mata Atlântica, que perfazem 490 ha $(21.7 \%$ da área total da Estação). Ao seu redor, predominam as culturas de cana-de-açúcar, eucalipto e gramíneas introduzidas para pastagens sobre latossolo roxo distrófico e eurotrófico. O fragmento estudado, de 74.47 ha, é cortado pela rodovia SP-333 que liga Sertãozinho à Barrinha.

A FSC está localizada entre $21^{\circ} 20^{\prime}$ e $21^{\circ}$ $27^{\prime} \mathrm{LS}$ e $47^{\circ} 18^{\prime}$ e $47^{\circ} 14^{\prime} \mathrm{LW}$, a uma altitude variando entre 540 e $944 \mathrm{~m}$. Ela é drenada por córregos e ribeirões, com ocorrência de cachoeiras e corredeiras, principalmente, no Rio Cubatão. O Rio Pardo marca o limite sul 
da fazenda e é o mais importante curso d'água da região. A vegetação é formada por bosques subtropicais semidecíduos que aparecem junto às serranias e galerias do Rio Cubatão e pequenos córregos. A área total da fazenda representa cerca de 6.300 ha. Os fragmentos de matas (cerca de 15) estão circundados por áreas dominadas pela cana-de-açúcar e por pastagens, sobre latossolos roxo e amarelo distrófico (Oliveira et al. 1983). O fragmento estudado (SI) tem 98.9 ha.

Ambas as áreas situam-se entre o clima tropical e tropical de altitude, com as estações do ano relativamente bem definidas. As chuvas iniciam-se em setembro, com a primavera e prolongam-se pelo verão (dezembro-fevereiro), demarcando as estações mais quentes e úmidas. O verão destaca-se pela maior abundância de chuvas, as quais diminuem no outono (março-abril). $\mathrm{O}$ inverno, de junho a agosto, é geralmente muito seco e frio e, às vezes, sem chuvas.

Amostragens. Os machos de Euglossini foram amostrados durante um ano, de novembro de 1988 a outubro de 1989, na EEZ e de abril de 1990 a março de 1991, na SI. As coletas foram feitas duas vezes por mês, das 08:00 às 17:00 horas, com uso de cineol, eugenol e vanilina, como iscas-odores. Quando da realização das coletas, chumaços de papel absorvente, um para cada isca, amarrados com barbante e pendurados nas ramagens de árvores, a uma altura de $1,5 \mathrm{~m}$ e distantes 12 $\mathrm{m}$ entre si, foram embebidos com os respectivos compostos a cada duas horas. Os machos atraídos foram capturados com rede entomológica, mortos em câmaras de $\mathrm{KCN}$ e acondicionados em sacos plásticos. O material coletado está depositado na Coleção Entomológica do Departamento de Biologia da F.F.C.L.R.P.- USP. Os dados climáticos, temperatura e precipitação pluviométrica, foram fornecidos pelas estações meteorológicas da EEZ e da Usina Amália (Município de Santa Rosa de Viterbo).

Análises dos Dados. Os índices de diversidade de espécies foram calculados pela função de Shannon-Wiener: $H^{\prime}=-\Sigma$ ph $\ln$ ph, onde ph é a proporção dos indivíduos pertencentes a h-ésima espécie e ln é o logaritmo neperiano (Pielou 1975). Os índices de equiidade foram calculados segundo Pielou (1966): J' = H'/H max, onde H' é o índice de Shannon-Wiener e H max é o logaritmo neperiano (ln) do número total de espécies na amostra; este índice varia de 0 a 1 . Para a medida de similaridade entre as áreas estudadas, no que diz respeito à composição de espécies, calculou-se o quociente de similaridade proposto por Sorensen (1948): Q.S. = $2 \mathrm{~J} / \mathrm{a}+\mathrm{b}$, onde $\mathrm{a}$ e b são os números de espécies que ocorrem nos habitats A e B e, J é o número de espécies presentes em ambos os habitats. Com o objetivo de se observar a semelhança entre as comunidades das áreas estudadas, não só em termos de composição de espécies, mas também considerando o número de indivíduos coletados, foi utilizado o cálculo da percentagem de similaridade (Southwood 1978): PS $=\Sigma$ da menor percentagem para cada espécie quando comparadas as duas áreas. Para a análise estatística dos índices de diversidade foi utilizado o "teste para diferença entre dois índices" (Zar 1984). Os padrões de distribuição das abundâncias das espécies ocorrendo nas duas áreas foram determinados de acordo com as metodologias propostas por Magurran (1988).

\section{Resultados}

Números de Espécies e de Indivíduos. $\mathrm{Na}$ SI, machos de 14 espécies foram atraídos pelos compostos aromáticos enquanto aqueles coletados na EEZ se distribuíram por 10 espécies (Tabela 1). Todas as espécies amostradas na EEZ foram encontradas na SI, proporcionando um coeficiente de similaridade alto (Q.S. $=0,83$ ) entre as áreas. Por outro lado, a percentagem de similaridade entre as áreas ( $\mathrm{PS}=52,9 \%)$ foi relativamente baixa. A maioria das espécies de Euglossini que compõem as comunidades em ambas as localidades foi do gênero Euglossa (11 espécies na SI e 7 na EEZ) (Tabela 1). Na SI, 
Tabela 1. Número de machos de Euglossini coletados no período de novembro de 1988 a outubro de 1989 na Estação Experimental de Zootecnia (EEZ), Sertãozinho, SP e de abril de 1990 a março de 1991 na Secção Itaoca (SI), Fazenda Santa Carlota, Cajuru, SP.

\begin{tabular}{lrr}
\hline & \multicolumn{2}{c}{ Número de machos } \\
\cline { 2 - 3 } Espécies de Euglossini & EEZ & SI \\
\hline & 4 & 12 \\
Euglossa (Glossura) annectans Dressler & 0 & 2 \\
Euglossa (Glossura) imperialis Cockerell & 5 & 2 \\
Euglossa (Euglossa) cordata (Linnaeus) & 51 & 232 \\
Euglossa (Euglossa) fimbriata Rebêlo \& Moure & 0 & 1 \\
Euglossa (Euglossa) leucotricha Rebêlo \& Moure & 1 & 18 \\
Euglossa (Euglossa) melanotricha Moure & 533 & 245 \\
Euglossa (Euglossa) pleosticta Dressler & 0 & 1 \\
Euglossa (Euglossa) securigera Dressler & 1 & 2 \\
Euglossa (Euglossa) townsendi Cockerell & 18 & 81 \\
Euglossa (Euglossa) truncata Rebêlo \& Moure & 0 & 1 \\
Euglossa (Euglossa) violaceifrons Rebêlo \& Moure & 24 & 19 \\
Eufriesea violacea (Mocsary) & 83 & 251 \\
Eulaema (Apeulaema) nigrita Lepeletier & 16 & 39 \\
Exaerete smaragdina (Guérin) & 736 & 906 \\
\hline Total & & \\
\hline
\end{tabular}

Euglossa fimbriata Rebêlo \& Moure, Euglossa pleosticta Dressler e Eulaema nigrita Lepeletier foram as mais abundantes, com seus machos correspondendo a $80,3 \%$ da amostra total; das demais espécies, a mais abundante foi Euglossa truncata Rebêlo \& Moure $(9,0 \%)$. Na EEZ a dominância foi de E. pleosticta $(72,4 \%)$, seguida por E. nigrita (11,3\%), E. fimbriata (6,9\%), Eufriesea violacea (Mocsary) (3,3\%), Exaerete smaragdina (Guérin) (2,2\%) e E. truncata $(2,4 \%)$; cada uma das outras espécies contribuiu com menos de 1,0\% (Tabela 1).

Os índices de diversidade, $\mathrm{H}^{\prime}=1.67$, e eqüidade, J' $=0,64$, obtidos para a amostra da SI, foram maiores que aqueles encontrados para a EEZ, H' $=1.03$ e J'=0.45. A baixa eqüidade na EEZ reflete a alta dominância de E. pleosticta, na amostra total. As distribuições das abundâncias relativas das espécies nas duas áreas (Fig. 1) foram descritas pelo modelo da série logarítmica $\left(\mathrm{X}^{2}=\right.$
8,32; $\mathrm{gl}=6 ; \mathrm{P}<0,05$, para SI e $\mathrm{X}^{2}=0,17 ; \mathrm{gl}$ $=3 ; \mathrm{P}<0,05$, para EEZ) com os valores do parâmetro $\alpha$ sendo 2,35 e 1,64 para as amostras da SI e EEZ, respectivamente.

Preferência por Compostos Aromáticos. $\mathrm{Na}$ EEZ, cineol atraiu $58.7 \%$ do total de machos capturados e 9 espécies; eugenol, atraiu 39,3\% e 6 espécies e vanilina atraiu 2,0\% e 4 espécies (Tabela 2). Na SI, a sequiência de importância dos compostos aromáticos foi a mesma, mas cineol foi proporcionalmente mais visitado (atraiu 71,5\% dos machos capturados e 13 espécies enquanto eugenol foi menos visitado pelos machos tendo atraído apenas $15,1 \%$ deles e 5 espécies, e vanilina foi o composto com menos ação atrativa (atraiu 13,4\% dos machos e 5 espécies).

Flutuação Sazonal. Em ambas as áreas os machos predominaram no outono $(53.8 \%$ da EEZ e $46.3 \%$ da SI), após o período mais 
Tabela 2. Número de machos de Euglossini capturados em cineol (C), eugenol (E) e vanilina (V) durante o período de novembro de 1988 a outubro de 1989 na Estação Experimental de Zootecnia (EEZ), Sertãozinho, SP e de abril de 1990 a março de 1991, na Secção Itaoca (SI), Fazenda Santa Carlota, Cajuru, SP.

\begin{tabular}{lrrrrrrrr}
\hline Espécies de & \multicolumn{3}{c}{ EEZ } & & & SI \\
Euglossini & $\mathrm{C}$ & $\mathrm{E}$ & $\mathrm{V}$ & Total & $\mathrm{C}$ & $\mathrm{E}$ & $\mathrm{V}$ & Total \\
\hline & 3 & 1 & 0 & 4 & 12 & 0 & 0 & 12 \\
E. annectans & 0 & 0 & 0 & 0 & 2 & 0 & 0 & 2 \\
E. imperialis & 5 & 0 & 0 & 5 & 2 & 0 & 0 & 2 \\
E. cordata & 50 & 1 & 0 & 51 & 221 & 8 & 3 & 232 \\
E. fimbriata & 0 & 0 & 0 & 0 & 1 & 0 & 0 & 1 \\
E. leucotricha & 1 & 0 & 0 & 1 & 16 & 2 & 0 & 18 \\
E. melanotricha & 259 & 268 & 6 & 533 & 174 & 37 & 34 & 245 \\
E. pleosticta & 0 & 0 & 0 & 0 & 1 & 0 & 0 & 1 \\
E. securigera & 0 & 1 & 0 & 1 & 2 & 0 & 0 & 1 \\
E. townsendi & 3 & 15 & 0 & 18 & 5 & 76 & 0 & 81 \\
E. truncata & 0 & 0 & 0 & 0 & 1 & 0 & 0 & 1 \\
E. violaceifrons & 17 & 0 & 7 & 24 & 0 & 0 & 19 & 19 \\
E. violacea & 82 & 0 & 1 & 83 & 199 & 0 & 52 & 251 \\
E. nigrita & 12 & 3 & 1 & 16 & 12 & 14 & 13 & 39 \\
E. smaragdina & 432 & 289 & 15 & 736 & 648 & 137 & 121 & 906 \\
\hline Total & 58.7 & 39.3 & 2.0 & 100 & 71.5 & 15.1 & 13.4 & 100 \\
\hline (\%) & & & & & & &
\end{tabular}

chuvoso do ano (de novembro a fevereiro) sendo menos abundantes no inverno $(0,7 \%$ da EEZ e $4,8 \%$ da SI), que corresponde ao período mais frio e seco do ano (Figs. 1,2). Das 10 espécies amostradas na EEZ apenas E. pleosticta e E. truncata tiveram machos

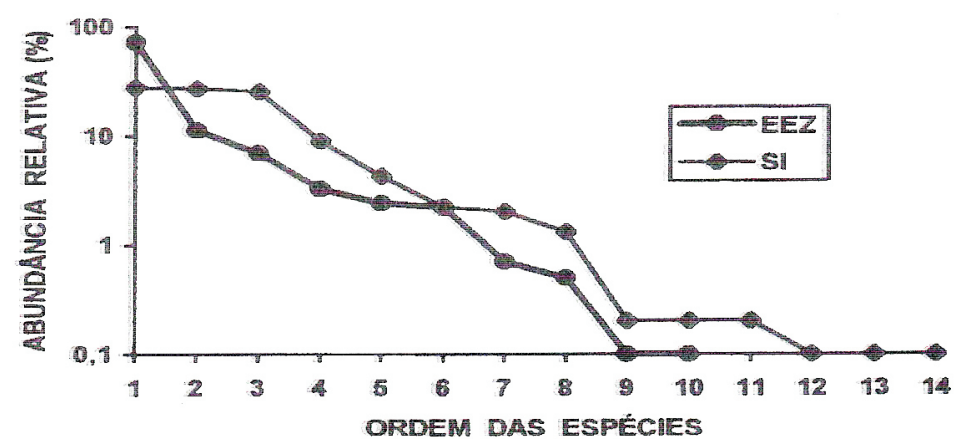

Figura 1. Curvas de abundância relativa de machos de Euglossini coletados no período de novembro de 1988 a outubro de 1989 na Estação Experimental de Zootecnia (EEZ), Sertãozinho, SP e de abril de 1990 a março de 1991 na Secção Itaoca (SI), Fazenda Santa Carlota, Cajuru, SP. Os pontos representam as espécies plotadas por suas abundâncias, como percentagem da amostra total, na seqüência da mais para a menos abundante. 
Tabela 3. Abundância sazonal e índices de diversidade (H') e eqüidade (J') das espécies de Euglossini amostradas durante o período de novembro de 1988 a outubro de 1989 na Estação Experimental de Zootecnia (EEZ), Sertãozinho, SP e de abril de 1990 a março de 1991 na Secção Itaoca (SI), Fazenda Santa Carlota, Cajuru, SP.

\begin{tabular}{|c|c|c|c|c|c|c|c|c|}
\hline Espécies de & & E E Z & & & & S I & & \\
\hline Euglossini & $\operatorname{Pr}$ & $\mathrm{Ve}$ & $\mathrm{Ou}$ & In & $\operatorname{Pr}$ & $\mathrm{Ve}$ & $\mathrm{Ou}$ & In \\
\hline E. annectans & 2 & 1 & 0 & 1 & 3 & 5 & 3 & 1 \\
\hline E. cordata & 0 & 0 & 5 & 0 & 0 & 2 & 0 & 0 \\
\hline E. fimbriata & 3 & 19 & 29 & 0 & 27 & 91 & 112 & 2 \\
\hline E. imperialis & 0 & 0 & 0 & 0 & 0 & 2 & 0 & 0 \\
\hline E. leucotricha & 0 & 0 & 0 & 0 & 1 & 0 & 0 & 0 \\
\hline E. melanotricha & 0 & 1 & 0 & 0 & 6 & 4 & 7 & 1 \\
\hline E. pleosticta & 56 & 167 & 307 & 3 & 41 & 95 & 101 & 8 \\
\hline E. townsendi & 0 & 1 & 0 & 0 & 0 & 2 & 0 & 0 \\
\hline E. truncata & 1 & 9 & 7 & 1 & 17 & 31 & 19 & 14 \\
\hline E. securigera & 0 & 0 & 0 & 0 & 1 & 0 & 0 & 0 \\
\hline E. violaceifrons & 0 & 0 & 0 & 0 & 0 & 1 & 0 & 0 \\
\hline E. violacea & 24 & 0 & 0 & 0 & 19 & 0 & 0 & 0 \\
\hline E. nigrita & 10 & 26 & 47 & 0 & 27 & 44 & 170 & 10 \\
\hline E. smaragdina & 0 & 15 & 1 & 0 & 3 & 14 & 21 & 1 \\
\hline Total de espécies & 6 & 8 & 6 & 3 & 10 & 11 & 7 & 7 \\
\hline Total de machos & 96 & 239 & 396 & 5 & 145 & 291 & 433 & 37 \\
\hline Diversidade $\left(\mathrm{H}^{\prime}\right)^{1}$ & $1,13 \mathrm{a}$ & $1,06 \mathrm{a}$ & $0,78 b$ & $0,95 \mathrm{ab}$ & $1,85 \mathrm{a}$ & $1,65 \mathrm{a}$ & $1,44 b$ & $1,50 \mathrm{ab}$ \\
\hline Eqüidade (J') & 0,63 & 0,51 & 0,44 & 0,86 & 0,81 & 0,69 & 0,74 & 0,77 \\
\hline
\end{tabular}

'Índices de diversidade seguidos da mesma letra, em cada uma das áreas estudadas, não diferem estatisticamente $(\mathrm{P}>0,05)$ pelo "teste para diferença entre dois índices" (Zar 1984)

coletados nas quatro estações do ano, sendo a primeira mais abundante no outono e a segunda no verão. E. fimbriata, E. nigrita, Euglossa annectans Dressler tiveram machos em atividade durante três estações, enquanto E. smaragdina ocorreu no verão e outono; e as demais espécies apenas em uma das estações (Tabela 3).

Na SI, 7 das 14 espécies amostradas apresentaram machos visitando as iscas em todas as estações; entre elas, E. fimbriata, Euglossa melanotricha Moure, E. pleosticta, E. nigrita e E. smaragdina apresentaram maior abundância no outono enquanto $E$. annectans e E. truncata, foram mais freqüentes no verão; as outras 7 espécies ocorreram em apenas uma estação do ano (Tabela 3). Embora o outono tenha sido a estação com maior abundância de machos em ambas as áreas, o verão foi a que apresentou o maior número de espécies amostradas. A abundância de machos e o número de espécies amostradas por estação não foram significantemente correlacionados em ambas as áreas $(\mathrm{r}=0,611$ e $\mathrm{r}=0,012, \mathrm{P}>0,05$, para a EEZ e SI, respectivamente). Tanto para a EEZ como para a SI nenhuma diferença estatisticamente significante $(P>0,05)$ foi encontrada entre os índices de diversidade da primavera, verão e inverno e entre aqueles do 


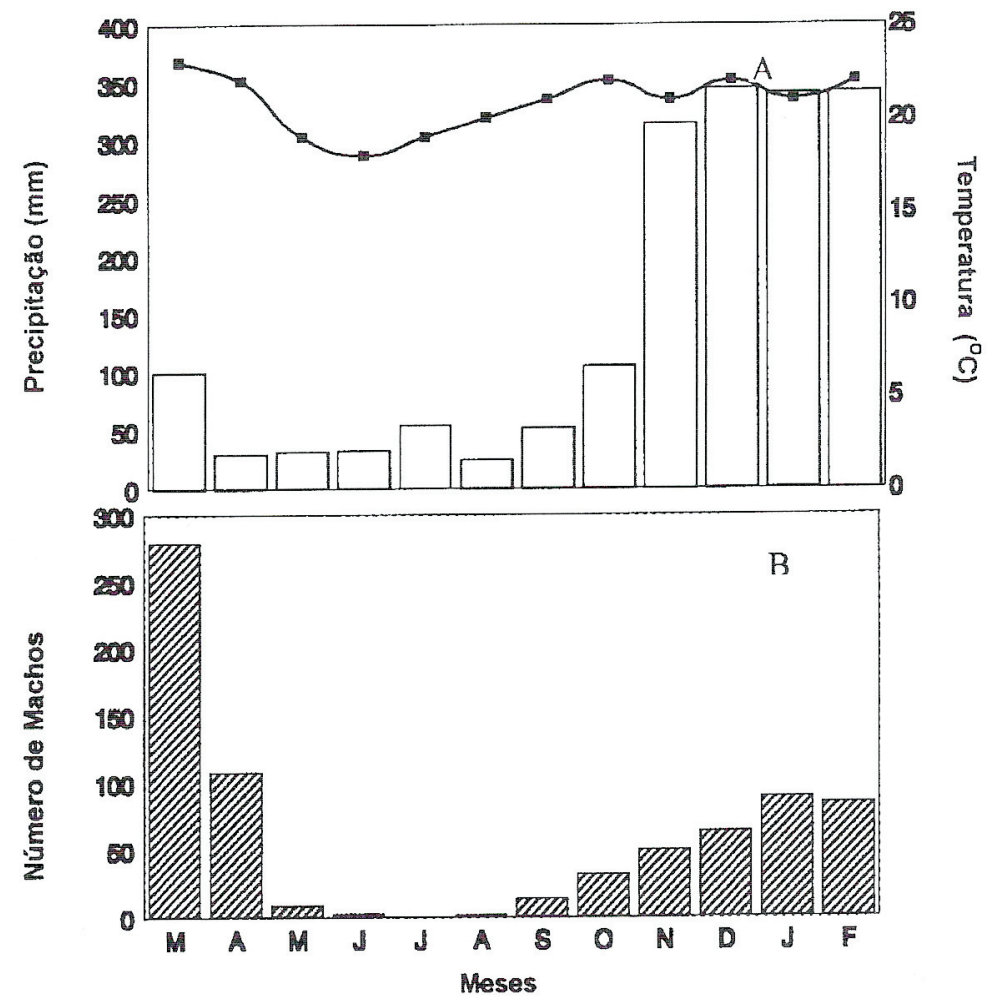

Figura 2. Condições climáticas (A) e número de machos de Euglossini (B) atraídos por iscas-odores, de novembro de 1988 a outubro de 1989, na Estação Experimental de Zootecnia, Sertãozinho, SP.

outono e inverno; por outro lado, a diversidade do outono diferiu significantemente $(\mathrm{P}<0,05)$ daqueles da primavera e verão. Os índices de equiidade das estações foram mais variáveis na EEZ e exceto o do inverno, os demais foram inferiores àqueles obtidos para a SI (Tabela 3).

\section{Discussão}

Rebêlo \& Garófalo (1991) estudaram, durante um ano, as populações de machos de Euglossini da Secção Santana (= SS) da FSC; na área estudada, uma capoeira em recuperação, distante cerca de $6 \mathrm{~km}$ da SI, registraram a ocorrência de 8 espécies atraídas por cineol, eugenol e vanilina. Nesse trabalho, realizado na mesma região do Estado, foram encontradas 14 espécies, 6 além daquelas amostradas na SS. Três daquelas 6 espécies, E. fimbriata, Euglossa leucotricha Rebêlo \& Moure e Euglossa violaceifrons Rebêlo \& Moure, são espécies novas (Rebêlo \& Moure 1995), confirmando a importância deste tipo de estudo.

O número de espécies foi maior na SI do que na EEZ e maior do que aquele relatado por Rebêlo \& Garófalo (1991) para a SS. Essas diferenças podem, simplesmente, refletir a ocorrência de algumas espécies em uma área e as suas ausências de outras. Assim, as 4 espécies amostradas na SI (Euglossa 
imperialis Cockerell, E. leucotricha, Euglossa securigera Dressler e E. violaceifrons) e ausentes na EEZ não foram, também, amostradas na SS. Contudo, considerando que nem sempre os compostos aromáticos selecionados para um estudo são atrativos para todas as espécies de uma área restrita (Ricklefs et al. 1969, Janzen et al. 1982, Ackerman 1983b, Oliveira \& Campos 1995), o fato daquelas 4 espécies terem sido representadas por apenas 1 ou 2 indivíduos pode indicar uma fraca associação delas com as iscas utilizadas. Isso, evidentemente, diminuiria a probabilidade da captura dos machos apesar deles estarem presentes na área. Por esse motivo, a ausência daquelas espécies nas amostragens da EEZ e SS deve ser analisada com precaução.

O padrão de abundância das espécies, nas duas áreas, com o predomínio daquelas com poucos indivíduos sobre as mais abundantes, foi similar àqueles encontrados por Ricklefs et al. (1969), Janzen et al. (1982), Ackerman (1983a) e Oliveira \& Campos (1995) como também em diversos outros trabalhos (Sakagami et al. 1967, Sakagami \& Fukuda 1973, Heithaus 1979, Laroca 1983, Pedro 1992, Camillo et al. 1995), realizados com outras metodologias, envolvendo comunidades dos Apoidea.

E. pleosticta, E. nigrita e E. fimbriata foram as espécies mais comuns nas duas áreas,. Na SI, elas são semelhantes quanto ao número de machos atraídos, apresentando cada uma delas, mais do dobro do somatório dos machos das 11 espécies restantes. Mas $E$. pleosticta continua sendo a espécie mais comum na região pois foi a mais abundante nas duas localidades deste estudo bem como na SS (Rebêlo \& Garófalo 1991). Na EEZ, E. pleosticta representou mais do dobro do total de machos das outras espécies juntas. É curioso a dominância de uma espécie solitária, nestas proporções. Diferenças neste nível geralmente são esperadas quando em estudos de comunidades ( Sakagami et al. 1967, Heithaus 1979, Laroca et al. 1982, Camargo \& Mazucato 1984, Silveira 1989, Bortoli \& Laroca 1990, Martins 1990, Pedro 1992,
Carvalho \& Bego 1995) são incluídas espécies sociais onde o número de indivíduos (operárias) em atividade de campo é muito grande. Se conhece muito pouco sobre a biologia de nidificação dos Euglossini (Garófalo 1994) mas, dificilmente se encontrará ninhos compartilhados por muitos adultos. Das informações disponíveis, o registro do maior número de fêmeas ativas em um ninho de Euglossini provem das observações de Pereira-Martins \& Kerr (1991). Esses autores relataram a participação de 24 fêmeas nas atividades de nidificação em um ninho de E. nigrita. Contudo, um número muito menor de fêmeas ativas em ninhos daquela espécie ocorre mais freqüentemente (Santos \& Garófalo 1994). Por outro lado, Garófalo et al. (1993) relataram que E. pleosticta foi a segunda espécie em frequiência de nidificação na SS da FSC; isto, seguramente, é conseqüência da abundância daquela espécie na região.

Embora os estudos utilizando iscas-odores na atração de machos de Euglossini propiciem informações importantes para o conhecimento do grupo certas espécies são bastante exigentes, quanto à sua ecologia, e raramente têm seus machos vistos visitando flores em áreas mais abertas ou sendo atraídos pelas iscas. Isso ocorre pela falta de associação com os compostos aromáticos utilizados ou por outras razões ainda desconhecidas. Por isso, tais espécies são omitidas das amostragens de trabalhos realizados em áreas restritas. Exemplo desses casos ocorrem na própria região desse estudo. Garófalo et al. (1993) encontraram dez ninhos de Eufriesea auriceps (Friese) estabelecidos em ninhos armadilhas colocados na SS da FSC indicando, assim, que tal espécie ocorre naquela região. Além disso, Garófalo et al. (não publicado) encontraram Exaerete dentata (Linnaeus) parasitando ninhos de E. auriceps encontrados, também, na FSC. Contudo, machos dessas duas espécies não foram atraídos pelas iscasodores. Assim, para se compreender melhor a dinâmica dessas abelhas e ter uma estimativa mais consistente a cerca da composição das comunidades de abelhas de uma determinada 


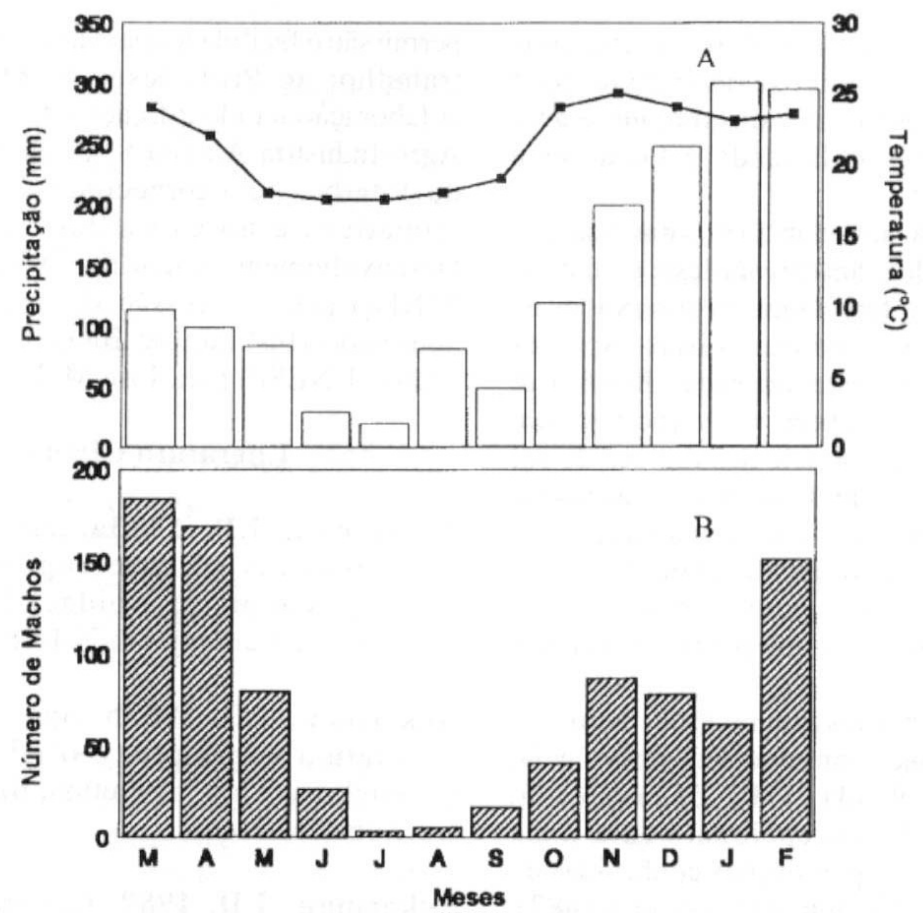

Figura 3. Condições climáticas (A) e número de machos de Euglossini (B) atraídos por iscas-odores, de abril de 1990 a março de 1991, na Secção Itaoca, Fazenda Santa Carlota, Cajuru, SP.

área, é aconselhável a obtenção de informações complementares, nas flores, nos ninhos, ou em outros locais.

Os compostos que têm sido utilizados na atração de machos de Euglossini no Estado de São Paulo, cineol, eugenol e vanilina, são, nesta ordem de importância, os únicos com ação efetiva sobre os machos (Rebêlo \& Garófalo 1991). Cineol atrai, diferencialmente, todas as espécies da região que se associam com iscas-odores, enquanto eugenol e vanilina atraem uma parcela menor delas, e E. truncata é a única espécie a mostrar nítida preferência por eugenol, embora se associe também com cineol e vanilina.

A abundância de muitas espécies de insetos tropicais muda sazonalmente (Wolda 1980). Para os Apoidea esta variação parece que é mais intensa nas florestas decíduas do que nas florestas úmidas (Janzen et al. 1982).
Nas florestas decíduas muitas espécies (p. ex. Centris spp., Gaesischia spp. - Apidae) voam e nidificam quase que exclusivamente na estação seca (Frankie \& Haber 1983), quando as árvores de florescimento sincrônico prevalecem (Frankie et al. 1983). Os Euglossini, no entanto, são mais abundantes e diversos durante a estação úmida, quando predominam as árvores que produzem flores dispersas (Frankie et al. 1983). Nas florestas úmidas, os Euglossini, machos e fêmeas, são vistos nas flores o ano todo, embora, apresentem respostas sazonais na abundância (Ackerman 1983a).

Nas florestas chuvosas da Costa Rica, os machos são menos ativos na estação seca, mas o mesmo número de espécies ocorre o ano inteiro enquanto nas florestas decíduas, eles são mais ativos na estação seca e as espécies mais ou menos constantes através do ano 
(Janzen et al. 1982). Nas florestas chuvosas do Panamá, os números de indivíduos e espécies são correlacionados durante o ano, sendo maiores no final da estação seca (Ackerman 1983a).

O padrão de abundância dos machos, nas florestas semidecíduas do nordeste do Estado de São Paulo, difere daqueles observados na América Central, porque várias espécies desaparecem na estação seca (Rebêlo \& Garófalo 1991). Embora o inverno não seja tão rigoroso como aquele das regiões do sul do Brasil ele é, geralmente, seco e, às vezes, com ausência de chuvas durante a estação. As maiores abundâncias de espécies e de indivíduos ocorrem durante o período mais quente e chuvoso, quando existe mais recursos disponíveis.

Muitas espécies de insetos tropicais apresentam pouca constância na abundância, entre os anos (Wolda 1980). Os Euglossini, no entanto, são excepcionalmente mais estáveis do que as populações conhecidas de outros insetos (Roubik \& Ackerman 1987). No presente estudo, a ordem de abundância das espécies não mudou significativamente nos anos de 1988-1989 e 1990-1991. As mesmas espécies dominaram nos dois locais de amostragem. Também não se detectou diferenças na preferência por compostos aromáticos.

Os Euglossini dos bosques subtropicais do nordeste do Estado de São Paulo constituem dois grupos fenológicos distintos. Um, representado por Eulaema, Euglossa e Exaerete, ocorrendo mais ou menos o ano todo e, o outro, representado por Eufriesea, um grupo altamente sazonal cujas espécies permanecem em atividade no campo de dois a cinco meses. Ambos padrões têm se mantido constante, nos anos sucessivos de estudos na região.

\section{Agradecimentos}

Os autores agradecem a família Sampaio Moreira, proprietária da Fazenda Santa Carlota, e à Direção da Estação Experimental de Zootecnia de Sertãozinho pela permissão e facilidades para a realização deste trabalho; ao Prof. Jesus S. Moure pela colaboração na identificação das espécies; à Agro-Indústria Amália S.A., de Santa Rosa do Viterbo, pelo fornecimento dos dados climáticos e ao Conselho Nacional de Desenvolvimento Científico e Tecnológico (CNPq) pela concessão de uma bolsa de doutorado (Instituto de Biociências de Rio Claro - UNESP) para José M. M. Rebêlo.

\section{Literatura Citada}

Ackerman, J.D. 1983a. Diversity and seasonality of male euglossine bees (Hymenoptera: Apidae) in Central Panama. Ecology 64: 274-283.

Ackerman, J.D. 1983b. Specificity and mutual dependency of the orchideuglossine bee interaction. Biol. J. Linn. Soc. 20:301-304.

Ackerman, J.D. 1989. Geographic and seasonal variation in fragrance choices and preferences of male euglossine bees. Biotropica 21: 340-347.

Becker, P., J.S. Moure \& F.J.A. Peralta. 1991. More about euglossine bees in Amazonian Forest Fragments. Biotropica 23: 586-591.

Bortoli, S. \& S. Laroca. 1990. Estudo biocenótico em Apoidea (Hymenoptera) de uma área restrita em São José dos Pinhais (PR., Sul do Brasil), com notas comparativas. Dusenia 15:1-112.

Camargo, J.M.F. \& M. Mazucato. 1984. Inventário da apifauna e flora apícola de Ribeirão Preto, SP. Brasil. Dusenia 14:5587.

Camillo, E., C.A. Garófalo, J.C. Serrano \& G. Muccillo. 1995. Diversidade e abundância sazonal de abelhas e vespas solitárias em ninhos armadilhas (Hymenoptera, Apocrita, Aculeata). Rev. 
Bras. Entomol. 39: 459-470.

Carvalho, A.M.C. \& L. R. Bego. 1995. Seasonality of dominant species of bees in the Panga Ecological Reserve, Cerrado, Uberlândia, MG. An. Soc. Entomol. Brasil 24: 329-337.

Dressler, R.L. 1982. Biology of the orchid bees (Euglossini). Ann. Rev. Ecol. Syst. 13: 373-394.

Frankie, G.H. \& W.A. Haber. 1983. Why bees move among mass-flowering neotropical trees, p. 361-372. In C. E. Jones \& R.J. Little (eds.). Handbook of Experimental Pollination Biology. New York. Van Nostrand Reinhold, Inc., 558 p.

Frankie, G.H., W.A. Haber, P.A. Opler, \& K.S. Bawa. 1983. Characteristics and organization of the large bee pollination system in the Costa Rican dry forest, $\mathrm{p}$. 411-447. In C.E. Jones \& R.J. Little (eds.) Handbook of Experimental Pollination Biology. New York. Van Nostrand Reinhold, Inc., 558 p.

Garófalo, C.A. 1994. Biologia de nidificação dos Euglossinae (Hymenoptera, Apidae). p. 17-26. In Anais do $1^{\circ}$ Encontro sobre abelhas, de Ribeirão Preto, SP., Brasil. $308 \mathrm{p}$.

Garófalo, C.A., E. Camillo, J.C. Serrano \& J.M.M. Rebêlo. 1993. Utilization of trap nest by Euglossini species (Hymenoptera: Apidae). Rev. Bras. Biol. 53:177-187.

Heithaus, E.R. 1979. Community structure of neotropical flower visiting bees and wasps: diversity and phenology. Ecology 60:190-202.

Hills, H.G., N.H. Williams, \& C.H. Dodson. 1972. Floral fragrance and isolating mechanisms in the genus Catasetum
(Orchidaceae). Biotropica 4: 61-76.

Janzen, D.H. 1971. Euglossine bees as longdistance pollinators of tropical plants. Science 171:203-205.

Janzen, D.H., P.J. Devries, M.L. Higgins, \& L.S. Kimsey. 1982. Seasonal and site variation in Costa Rican euglossine bees at chemical baits in lowland deciduous and evergreen forests. Ecology 63: 6674.

Kimsey, L.S. 1987. Generic relationships within the Euglossini (Hymenoptera: Apidae). Syst. Ent. 12:63-72.

Laroca, S. 1983. Biocenotics of wild bees (Hymenoptera, Apoidea) at three neartic sites, with comparative notes on some neotropical assemblages. Tese de doutorado. University of Kansas, USA, $194 \mathrm{p}$.

Laroca, S., J.R. Cure \& C. Bortoli. 1982. A associação das abelhas silvestres (Hymenoptera, Apoidea) de uma área restrita no interior da cidade de Curitiba (Brasil): uma abordagem biocenótica. Dusenia 13:93-117.

Magurran, A.E. 1988. Ecological diversity and its measurements. New Jersey, Princeton University Press, Princeton, $179 \mathrm{p}$.

Martins, C.F. 1990. Estrutura da comunidade de abelhas (Hym., Apoidea) na caatinga (Casa Nova, BA) e na Chapada Diamantina (Lençóis, BA). Tese de doutorado. Instituto de Biociências-USP, São Paulo, 159 p.

Moure, J.S. 1967. A check-list of the know euglossine bees (Hymenoptera, Apidae). Atas Simpos. Biota Amazôn. 5:395-415.

Oliveira, M.L. \& L.A.O. Campos. 1995. Abundância, riqueza e diversidade de 
abelhas Euglossinae (Hymenoptera, Apidae) em florestas contínuas de terra firme na Amazônia Central, Brasil. Rev. Bras. Zool. 12: 547-556.

Oliveira, V., A.M. Costa \& W.P. Azevedo. 1983. Pedologia, Folhas SF.23/24., Rio de Janeiro. In: Projeto Radam Brasil, Levantamento de Recursos Naturais 32: 385-552.

Pearson, D.L. \& R.L. Dressler. 1985. Two year study of male orchid bee (Hymenoptera: Apidae: Euglossini) attraction to chemical baits in lowland south-eastern Peru. J. Tropical Ecol. 1: 37-54.

Pedro, S.R.M. 1992. Sobre as abelhas (Hymenoptera, Apoidea) em um ecossistema de Cerrado (Cajuru, NE do Estado de São Paulo): composição, fenologia e visita às flores. Tese de mestrado. FFCLRP-USP, Ribeirão Preto, $231 \mathrm{p}$.

Pereira-Martins, S.R. \& W.E. Kerr. 1991. Biologia de Eulaema nigrita. 1. Construção de células, oviposição e desenvolvimento. Pap. Avul. Zool. 37:227-235.

Pielou, E.C. 1966. An introduction to mathematical ecology. New York, John Wiley \& Sons. 286 p.

Pielou, E.C. 1975. Ecological diversity. New York, John Wiley \& Sons. 165 p.

Rebêlo, J.M.M. 1990. Diversidade, sazonalidade e preferência por iscasodores de machos de Euglossini (Hymenoptera, Apoidea, Apidae). Tese de mestrado. FFCLRP-USP, Ribeirão Preto, 104 p.

Rebêlo, J.M.M. \& C.A. Garófalo. 1991. Diversidade e sazonalidade de machos de Euglossini (Hymenoptera, Apidae) e preferências por iscas-odores em um fragmento de floresta no sudeste do Brasil. Rev. Brasil. Biol. 51: 787-799.

Rebêlo, J.M.M. \& J.S. Moure. 1995. As espécies de Euglossa Latreille do Nordeste de São Paulo (Apidae, Euglossinae). Rev. Bras. Zool. 12:445466.

Ricklefs, R.E., R.M. Adams \& R.L. Dressler. 1969. Species diversity of Euglossa in Panama. Ecology 50: 713716.

Roubik, D.W. \& J.D. Ackerman. 1987. Long-term ecology of euglossine orchidbees (Apidae: Euglossini) in Panama. Oecologia 73: 321-333.

Sakagami, S.F., S. Laroca \& J.S. Moure. 1967. Wild bee biocenotics in São José dos Pinhais (PR), South Brazil. Preliminary Report. J. Fac. Sci. Hokkaido Univ. VI Zool. 16:253-291.

Sakagami, S.F. \& H. Fukuda. 1973. Wild bee survey at the Campus of Hokkaido University. J. Fac. Sci. Hokkaido Univ. VI Zool. 19:190-250.

Santos, M.L. \& C.A. Garófalo. 1994. Nesting biology and nest re-use of Eulaema nigrita (Hymenoptera, Apidae, Euglossini). Ins. Soc.41:99-110.

Silveira, F.A. 1989. Abelhas silvestres (Hymenoptera: Apoidea) e suas fontes de alimento no cerrado da Estação Florestal de Experimentação de Paraopeba-MG. Tese de mestrado. UFV. Viçosa, 50 p.

Sorensen, T. 1948. A method of establishing group of equal amplitude in plant sociology based on similarity of species content and its application to analyses of the vegetation on Danish commons. Biol. Skr. 5:1-34. 
Southwood, T.R.E. 1978. Ecological methods with particular reference to the study of Insect Populations. 2nd ed., New York, John Wiley \& Sons, Inc. 524 p.

Zar, J.H. 1984. Bioestatistical analysis. 2nd ed., New Jersey, Prentice-Hall, Inglewood Cliffs. 718 p.

Williams, N.H. 1982. The biology of orchids and euglossine bees, p. 119-171. In J. Arditti (ed.), Orchid biology: reviews and perspectives,II. Ithaca, NY, Cornell Univ. Press.
Williams, N.H. \& Witthen, W.M. 1983. Orchid floral fragrance and male euglossine bees. Methods and advances in the last sesquidecade. Biol. Bull. 164:355-395.

Wolda, H. 1980. Seasonality of tropical insects. I. Leafhoppers (Homoptera) in Las Cumbres, Panama. J. Anim. Ecol. 49: 277-290.

Recebido em 24/10/96. Aceito em 10/06/97. 